\title{
BMJ Open Ability of plasma-based or serum-based assays of D-dimer and FDP for diagnosing periprosthetic joint infection: protocol for a prospective single-centre, parallel comparative study
}

Hong Xu, ${ }^{1}$ Jinwei Xie, ${ }^{1}$ Jing Zhou, ${ }^{2}$ Xing Wang, ${ }^{1}$ Duan Wang, ${ }^{1}$ Zeyu Huang (D) , Zongke Zhou (D) ${ }^{1}$

To cite: Xu H, Xie J, Zhou J, et al. Ability of plasma-based or serum-based assays of Ddimer and FDP for diagnosing periprosthetic joint infection: protocol for a prospective single-centre, parallel comparative study. BMJ Open 2021;11:e046442. doi:10.1136/ bmjopen-2020-046442

- Prepublication history for this paper is available online. To view these files, please visit the journal online (http://dx.doi. org/10.1136/bmjopen-2020046442).

HX, JX and JZ contributed equally.

The study was performed in Department of Orthopaedic Surgery, West China Hospital, Sichuan University.

$\mathrm{HX}, \mathrm{JX}$ and $\mathrm{JZ}$ are joint first authors.

Received 31 October 2020 Accepted 08 June 2021

Check for updates

(c) Author(s) (or their employer(s)) 2021. Re-use permitted under CC BY-NC. No commercial re-use. See rights and permissions. Published by BMJ.

For numbered affiliations see end of article.

Correspondence to

Dr Zongke Zhou;

zongkeHX@163.com

\section{ABSTRACT}

Introduction Several studies have evaluated the diagnostic values of $D$-dimer and fibrin degradation product (FDP) for detecting periprosthetic joint infection (PJI), but their conclusions have been inconsistent. More importantly, whether the diagnostic value of these two biomarkers differs depending on whether they are assayed in plasma or serum is unclear.

Methods and analysis This prospective single-centre, parallel comparative study will involve patients planning to undergo revision hip or knee arthroplasty due to an aseptic mechanical failure or PJI. After the patients provide written informed consent, their serum and plasma will be sampled and assayed for D-dimer and FDP. The diagnostic value of these two biomarkers in plasma and serum will be compared with one another and with the value of two traditional inflammatory markers ( $C$ reactive protein and erythrocyte sedimentation rate) used to diagnose PJI according to the 2013 International Consensus Meeting criteria. Diagnostic value will be assessed in terms of area under receiver operating characteristic curves, sensitivity, specificity, as well as positive and negative predictive values, all calculated based on the optimal cut-off determined from the Youden index.

Ethics and dissemination This study was approved by the Ethics Committee of our hospital (approval no. 2020-859). Written informed consent will be obtained from all patients before enrollment. Study findings will be disseminated widely through peer-reviewed publications and conference presentations.

Trial registration number This study was registered in the Chinese Clinical Trial Registry (ChiCTR2000038547), and it is ongoing.

Title:

\section{INTRODUCTION}

Periprosthetic joint infection (PJI) is a catastrophic complication after total hip arthroplasty (THA) or total knee arthroplasty (TKA). It increases hospitalisation costs, prolongs treatment and increases risk of morbidity and mortality. ${ }^{12}$ PJI
Strengths and limitations of this study

- This is a prospective study with enough sample size.

- All related indicators used to diagnose periprosthetic joint infection will be collected.

- This is a single-centre study.

- The diagnostic value of enrolled biomarkers for the patients with inflammatory arthritis and venous thromboembolic disease needed to be further evaluated in the future.

incidence is increasing with the growing number of primary total joint arthroplasties (TJAs). ${ }^{3}{ }^{4}$ Infection should be detected as early as possible, and certainly before revision surgeries, because it substantially affects planning of treatment and management aimed at preserving the implanted prosthesis and joint function. Detection of PJI is also important for managing patients' expectations. ${ }^{5}$

One possibility for diagnosing PJI is to assay blood or synovial fluid for appropriate biomarkers. ${ }^{6}$ Blood tests on patients with suspected infection are important because they can be rapidly performed in advance of joint aspiration. A reliable, sensitive biomarker can reduce the rate of missed infections, but the two inflammatory biomarkers currently recommended for diagnosing PJI according to the 2013 International Consensus Meeting (ICM) criteria, C reactive protein (CRP) and erythrocyte sedimentation rate (ESR), are not highly sensitive. Both indices can be normal in infections of certain weakly virulent pathogens, such as Propionibacterium acnes. ${ }^{6-8}$

Alternative biomarkers for diagnosing PJI from blood tests may be D-dimer and fibrin degradation product (FDP), both products of fibrinolysis. ${ }^{910}$ Levels of D-dimer in plasma have already proven to be a useful marker 
of lethality for very early-stage blood-borne infections. ${ }^{11}$ Several studies subsequently reported the diagnostic values of D-dimer and FDP for diagnosis PJI, ${ }^{9} 1213$ though currently these biomarkers are used in the clinic primarily to exclude venous thromboembolism ${ }^{14}$ and monitor postoperative fibrinolysis. ${ }^{15}$ And the 2018 revision of ICM criteria had recommended the levels of serum D-dimer as a reference biomarker for diagnosing PJI alongside CRP. $^{16}$

Unfortunately, the evidence supporting D-dimer levels as a biomarker of PJI may not be entirely reliable. ${ }^{17}$ Studies have reported inconsistent findings about its diagnostic performance, ${ }^{101318}$ and all but one of these studies ${ }^{19}$ were retrospective. In addition, the D-dimer was assayed in serum in some studies ${ }^{918} 2021$ but from plasma in others. ${ }^{10}{ }^{13}$ More importantly, as suggested by Pannu et $a l,{ }^{22}$ some authors of the published studies may have failed to notice the difference between plasma and serum and caused some mistakes in their papers. But several meta-analyses have already been performed to compared the diagnostic values of D-dimer between plasma and serum, which may lead to more confusion. ${ }^{23-25}$ In addition, although some studies have demonstrated that serum D-dimer is superior to plasma D-dimer in diagnosis of PJI, ${ }^{23}{ }^{26}$ we are unaware of studies comparing the diagnostic value of D-dimer levels in plasma or serum sampled from the same patients. Consequently, high-quality prospective studies that address these research gaps are needed to validate the use of D-dimer as a PJI biomarker.

In addition to D-dimer, FDP is also the product of fibrinolysis. Fujimoto et $a l^{27}$ reported that increased serum FDP level prevent the negative conversion of CRP value after TKA. Plasma levels of FDP are also strongly related to inflammatory responses after TJA. ${ }^{15}$ Our previous retrospective study has called into question whether plasma levels of FDP can reliably diagnose PJI, ${ }^{10}$ but these results should be confirmed in prospective analysis.
Furthermore, we are unaware of studies assessing the potential of serum levels of FDP to diagnose PJI.

Therefore, we plan a prospective study to address the following three questions: (1) whether there is a difference between serum and plasma D-dimer or FDP in patients with or without inflammatory diseases such as rheumatoid arthritis (RA) and ankylosing spondylitis (AS) before primary TJA; more importantly, (2) whether there is a difference between serum and plasma D-dimer or FDP for identifying PJI before revision hip or knee arthroplasty and (3) whether D-dimer and FDP can improve the diagnostic values of CRP and ESR in PJI.

\section{METHODS \\ Study design}

This will be a prospective single-centre, parallel comparative study to evaluate and compare the diagnostic performance of D-dimer and FDP assayed in serum and plasma. This study will include (1) two control groups of patients who are planning to undergo primary TKA or THA with or without inflammatory diseases, respectively, (2) a non-PJI group of patients planning to undergo revision arthroplasty due to an aseptic mechanical failure and without infection and (3) a PJI group of patients planning to undergo revision arthroplasty due to infection as defined by 2013 ICM criteria (table 1). ${ }^{28} 29$

\section{Participants and eligibility criteria}

Patients who have been diagnosed with primary osteoarthritis of the knee or hip, osteonecrosis of the femoral head or developmental dysplasia of the hip and who are planning to undergo primary TKA or THA will be enrolled as group A. And patients who have been diagnosed with RA or AS of the knee or hip and who are planning to undergo primary TKA or THA will be enrolled as group B. These two groups of patients will be analysed

Table 12013 ICM criteria for diagnosing PJI and the thresholds of the minor diagnostic criteria

\begin{tabular}{|c|c|c|c|}
\hline & & \multicolumn{2}{|l|}{ Recommended threshold } \\
\hline & Diagnostic criteria & Acute (<90 days) & Chronic (>90 days) \\
\hline Major criteria & $\begin{array}{l}\text { Two positive periprosthetic } \\
\text { cultures with phenotypically identical } \\
\text { microorganisms or } \\
\text { A sinus tract communicating with the } \\
\text { joint }\end{array}$ & - & - \\
\hline
\end{tabular}

$\mathrm{PJI}$ is present when one of the major criteria, or three of five of the minor criteria are met.

CRP, C reactive protein; ESR, erythrocyte sedimentation rate; ICM, International Consensus Meeting; PJI, periprosthetic joint infection;

PMN\%, polymorphonuclear neutrophil percentage; SF, synovial fluid; WBC, white blood cell. 
to examine differences between serum- and plasma-based assays of D-dimer and FDP, as well as the effect of inflammatory diseases on their levels. Another two groups of patients who plan to undergo revision hip or knee arthroplasty due to an aseptic mechanical failure (group C) or infection (group D) will be analysed to assess the ability of D-dimer and FDP in serum and plasma to diagnose PJI. Furthermore, subgroups will be designed to explore the effect of comorbidities, for example, tumours, on the diagnostic values of serum and plasma D-dimer and FDP for identifying PJI.

Patients will be excluded if they (1) refuse to participate in the study, (2) have been diagnosed with deep vein thrombosis, (3) are taking oral anticoagulants or antiplatelet drugs, (4) have haemophilia or (5) use antibiotics within 2 weeks before operation.

\section{PJI diagnostic criteria and outcome evaluation}

PJI will be defined in this study according to the 2013 ICM criteria. ${ }^{28}$ Patients who plan to undergo revision hip or knee arthroplasty will be divided into those who are infected or not according to the criteria.

After obtaining written informed consent from enrolled patients, nurses will collect fasting venous blood samples on the day of admission. The samples will be sent to our hospital's clinical laboratory within 1-2 hour. D-dimer and FDP levels in plasma and serum will be assayed separately by immunoturbidimetry using an automatic coagulation analyser (CS-5100; Japan) and commercial test kit (Siemens), respectively. In addition, routine blood examination, serum CRP and ESR will be tested at the same time.

The affected joint in each patient planning to undergo revision arthroplasty will be aspirated on the day of admission or on the following day. The aspirated synovial fluid will be sent immediately for determination of white blood cell count and differential neutrophil count, polymorphonuclear neutrophil percentage as well as aerobic and anaerobic cultures with blood culture bottles adapted to bacterial and fungal growth. If the preoperative samples of aspirated joint fluid are insufficient for culturing, the joint cavity will be flushed with aseptic saline, which will be sent for cultures. In addition, synovial fluid will be collected intraoperatively during revision arthroplasty and sent for cultures. Cultures will be maintained for 5 days routinely, but then it will be extended to 21 days if preoperative cultures have proven to be negative and there is a high clinical suspicion for infection. Fungal and mycobacterial cultures will be performed only in higher risk scenarios. Four or more periprosthetic tissues will be biopsied during all revision procedure and sent for histology analysis and cultures. And the neutrophils count of these tissues will be determined using microscope.

\section{Outcomes and data collection}

Baseline data will be recorded about age, sex, diagnosis at discharge, comorbidities including hypertension, diabetes, chronic obstructive pulmonary disease, coronary heart disease, inflammatory diseases and malignant tumours; results of laboratory tests of blood and synovial fluid, culture results of synovial fluid and histology as well as culture results of periprosthetic tissues. Data will also be recorded about the presence of comorbidities such as RA and AS that may elevate the levels of inflammatory or fibrinolytic biomarkers.

\section{Sample size calculation}

MedCalc V.12.7 (MedCalc Software bvba, Ostend, Belgium) was used to calculate sample size. Previous work showed the area under the receiver operating characteristic curve (AUC) for plasma levels of D-dimer to be $0.657,{ }^{13}$ much lower than the AUC of serum D-dimer reported by Shahi et at; therefore, the AUC of plasma D-dimer was used to calculate the sample size with type I (significant) error set to 0.05 and type II (1-power) error set to 0.2 . Taking into account the ratio of 1.24 for noninfected patients to infected patients undergoing revision arthroplasty at our hospital, ${ }^{2}$ we calculated minimal sample sizes of 59 non-infected patients and 47 infected patients. And the patients who are planning to undergo primary TKA or THA with or without inflammatory diseases will be enrolled 40 patients, respectively. Consequently, a total of about 186 patients will be enrolled in four groups (figure 1).

\section{Statistical analyses}

All data will be entered in a timely fashion by two trained researchers working independently. Descriptive statistics will be presented as mean (SD) for normally distributed continuous variables or median (IQR) for non-normally distributed data, and frequency (percentage) for categorical variables. Student's t-test will be used to analyse the normal distributed numerical variables; while Wilcoxon Mann-Whitney $\mathrm{U}$ test will be used to analyse the numerical variables with non-normal distribution or unequal variance; Pearson $\chi^{2}$ test and Fisher exact test will be used to analyse the qualitative variables. Differences associated with $\mathrm{p}<0.05$ are considered significant.

Receiver operating characteristic curves will be calculated to examine the relationship between the rate of true positives (sensitivity) and the rate of false positives (1-specificity), and to determine the AUC. The Youden index will be used to determine the optimal predictive cut-offs for calculating AUC. Diagnostic performance of D-dimer and FDP from plasma or serum will be assessed in terms of AUC, sensitivity, specificity, positive and negative predictive values. And the z-test will be used to compare AUCs for tested biomarkers. Statistical analyses will be performed using SPSS V.24 (IBM, Armonk, NY, USA) and MedCalc V.12.7 (MedCalc Software bvba).

\section{Study start and duration}

The study is ongoing. Our hospital is a designated national centre for the diagnosis and treatment of complicated and critical diseases and annually $>3000$ TJA procedures are performed there. Therefore, the required number of 


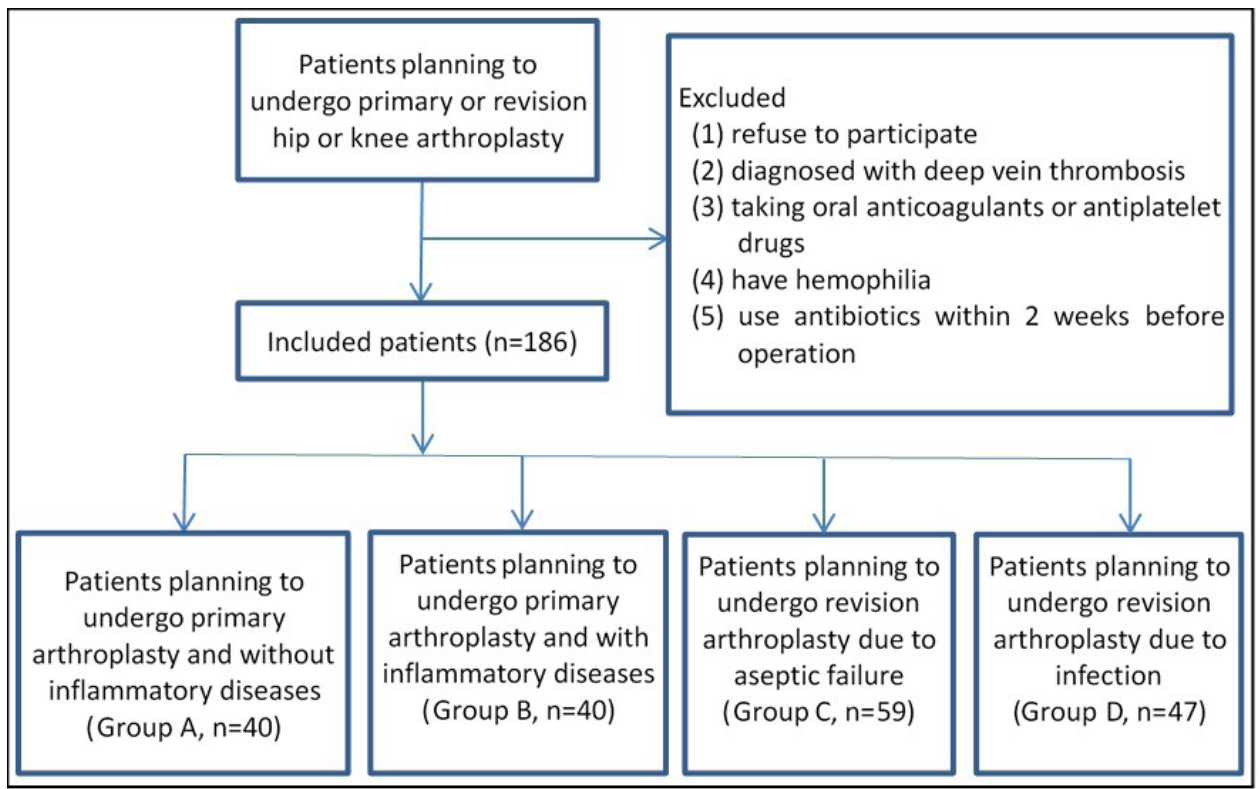

Figure 1 Flow diagram of the study design.

subjects is likely to be enrolled during the 1-2 years. And this study started in October 2020 and planned to end in October 2021.

\section{DISCUSSION}

Although the potential of D-dimer and FDP for identifying PJI has been evaluated in recent years, nearly no studies offering high-level, prospective evidence have been conducted. In addition, it is unclear whether levels of these biomarkers in serum or plasma may be more clinically useful. The purpose of this prospective study is to provide high-quality evidence for determining whether D-dimer and/or FDP levels in plasma and/or serum can be accurate and reliable diagnostic biomarkers for PJI. In this way, the results of this study will validate or refute the recommendations concerning the use of serum D-dimer in 2018 ICM criteria. The results will be the most extensive prospective data so far on the diagnostic potential of D-dimer and FDP for PJI, and apparently the first direct comparison between serum-based and plasma-based assays of these biomarkers.

\section{Strengths of this study}

This study will be only the second prospective analysis, aside from one previous work, ${ }^{19}$ to assess diagnostic performance of D-dimer or FDP. More importantly, the study will assay plasma and serum from the same patients simultaneously, which has rarely been done previously. This study may allow definitive conclusions about the diagnostic value of these biomarkers.

Furthermore, retrospective studies on D-dimer usually did not assay synovial fluid and periprosthetic tissues in patients undergoing revision arthroplasty when infection was not suspected. However, these tests are important for detecting infections caused by some weakly virulent pathogens that cannot elevate PJI-related biomarkers such as
CRP or ESR and without causing obvious clinical manifestations such as pain or fever. ${ }^{3031}$ As a further measure to detect infections, this study will include repeated preoperative joint aspiration of each patient planning to undergo revision arthroplasty, and synovial fluid and periprosthetic tissues will also be collected during surgery and tested to avoid missing some infected subjects.

\section{Limitations of this study}

First, though previous studies have shown that patients with inflammatory arthritis such as RA and AS are at higher risk of infection than those without it, detecting infection in those patients after TJA remains challenging because traditional biomarkers such as CRP and ESR can be unreliable for those patients. ${ }^{32} 33$ Although our study may help address this problem, it may not lead to definitive conclusions about the diagnostic performance of D-dimer and FDP because of the limited sample size. Second, to reduce confounding in our analysis, we will exclude patients with deep vein thrombosis or haemophilia, as well as patients taking oral anticoagulants or antiplatelet drugs. Thus, the generalisability of our findings for those patient subgroups will be unclear. Third, we will exclude the patients if they use antibiotics within 2 weeks before operation, since it can decrease the diagnostic value of CRP and ESR, and the positive rate of synovial fluid culture ${ }^{3435}$ hence, the use of our findings for those patients will be uncertain. Even so, we believe this study could provide significant information about the diagnostic values of D-dimer and FDP from both serum and plasma for identifying PJI.

\section{Data sharing plan}

The data from this study that are needed to replicate the main findings will be made available by the principal investigators on reasonable request. 


\section{ETHICS AND DISSEMINATION}

This study was approved by the Ethics Committee of our hospital (approval no. 2020-859), and it will be carried out in accordance with the published study protocol and with the latest version of the Declaration of Helsinki. Written informed consent will be obtained from all patients before enrollment. The results of this study will be distributed through publication in a peer-reviewed research journal and conference presentations.

\section{Author affiliations}

${ }^{1}$ Department of Orthopaedic Surgery, Sichuan University West China Hospital, Chengdu, Sichuan, China

${ }^{2}$ Department of Laboratory Medicine, Sichuan University West China Hospital, Chengdu, Sichuan, China

\section{Twitter Zeyu Huang @Dr_ZeYu_Huang}

Acknowledgements We thank all authors of included studies. We also thank $\mathrm{A}$ Chapin Rodríguez, PhD, from Creaducate Enterprises Ltd for editing the English text of a draft of this manuscript.

Contributors $\mathrm{HX}$ and JX screened the literatures, drafted the work and revised it critically for important intellectual content. JZ and XW designed the trial and modified the study scheme. DW and ZYH participated in final approval of the version to be published. ZZ contributed to the conception and design of the work and revised the manuscript. All authors have given their final approval of the version to be published and agree to be accountable for all aspects of the work.

Funding This study is supported by the Key Research \& Development Program of Science \& Technology Department of Sichuan Province (No. 2018SZ0255), the 1.3.5 Project for Disciplines of Excellence, West China Hospital, Sichuan University (No. ZYJC18039).

Disclaimer The funding bodies have no authority over the design of the study; sample collection or storage; data collection, management, analysis or interpretation; or manuscript drafting or revision.

Competing interests None declared.

Patient and public involvement Patients and/or the public were not involved in the design, or conduct, or reporting, or dissemination plans of this research.

Patient consent for publication Not required.

Provenance and peer review Not commissioned; externally peer reviewed.

Open access This is an open access article distributed in accordance with the Creative Commons Attribution Non Commercial (CC BY-NC 4.0) license, which permits others to distribute, remix, adapt, build upon this work non-commercially, and license their derivative works on different terms, provided the original work is properly cited, appropriate credit is given, any changes made indicated, and the use is non-commercial. See: http://creativecommons.org/licenses/by-nc/4.0/.

\section{ORCID iDs}

Zeyu Huang http://orcid.org/0000-0002-0456-8379

Zongke Zhou http://orcid.org/0000-0002-9037-4756

\section{REFERENCES}

1 Parvizi J, Shohat N, Gehrke T. Prevention of periprosthetic joint infection: new guidelines. Bone Joint J 2017;99-B:3-10.

$2 \mathrm{Xu} \mathrm{H,} \mathrm{Xie} \mathrm{J,} \mathrm{Yang} \mathrm{J.} \mathrm{Plasma} \mathrm{fibrinogen} \mathrm{and} \mathrm{platelet} \mathrm{count} \mathrm{are}$ Referable tools for diagnosing periprosthetic joint infection: a singlecenter retrospective cohort study. J Arthroplasty 2020;35:1361-7.

3 Klug A, Gramlich Y, Rudert M, et al. The projected volume of primary and revision total knee arthroplasty will place an immense burden on future health care systems over the next 30 years. Knee Surg Sports Traumatol Arthrosc 2020. doi:10.1007/s00167-020-06154-7. [Epub ahead of print: 15 Jul 2020].

4 Cozzi Lepri A, Del Prete A, Soderi S, et al. The identification of pathogens associated with periprosthetic joint infection in two-stage revision. Eur Rev Med Pharmacol Sci 2019;23:101-16.

5 Fehring TK, Odum SM, Berend KR, et al. Failure of irrigation and débridement for early postoperative periprosthetic infection. Clin Orthop Relat Res 2013;471:250-7.
6 Parvizi J, Gehrke T, Chen AF. Proceedings of the International consensus on periprosthetic joint infection. Bone Joint $J$ 2013;95B:1450-2.

7 Kanafani ZA, Sexton DJ, Pien BC, et al. Postoperative joint infections due to Propionibacterium species: a case-control study. Clin Infect Dis 2009;49:1083-5.

8 Kheir MM, Tan TL, Shohat N, et al. Routine diagnostic tests for periprosthetic joint infection demonstrate a high false-negative rate and are influenced by the infecting organism. J Bone Joint Surg Am 2018;100:2057-65.

9 Shahi A, Kheir MM, Tarabichi M, et al. Serum D-dimer test is promising for the diagnosis of periprosthetic joint infection and timing of reimplantation. J Bone Joint Surg Am 2017;99:1419-27.

$10 \mathrm{Xu} \mathrm{H}$, Xie J, Huang Q, et al. Plasma fibrin degradation product and Ddimer are of limited value for diagnosing periprosthetic joint infection. J Arthroplasty 2019;34:2454-60.

11 Schwameis M, Steiner MM, Schoergenhofer C, et al. D-Dimer and histamine in early stage bacteremia: a prospective controlled cohort study. Eur J Intern Med 2015;26:782-6.

12 Klim SM, Amerstorfer F, Gruber G. Fibrinogen - a practical and cost efficient biomarker for detecting periprosthetic joint infection. Sci Rep 2018;8:8802.

13 Li R, Shao H-Y, Hao L-B, et al. Plasma fibrinogen exhibits better performance than plasma D-dimer in the diagnosis of periprosthetic joint infection: a multicenter retrospective study. J Bone Joint Surg Am 2019;101:613-9.

14 den Exter PL, Klok FA, Huisman MV. Diagnosis of pulmonary embolism: advances and pitfalls. Best Pract Res Clin Haematol 2012;25:295-302.

15 Lei Y, Xie J, Xu B, et al. The efficacy and safety of multipledose intravenous tranexamic acid on blood loss following total knee arthroplasty: a randomized controlled trial. Int Orthop 2017;41:2053-9.

16 Parvizi J, Tan TL, Goswami K, et al. The 2018 definition of periprosthetic hip and knee infection: an evidence-based and validated criteria. J Arthroplasty 2018;33:1309-14.

17 Pearson LN, Moser KA, Schmidt RL. D-Dimer varies widely across instrument platforms and is not a reliable indicator of periprosthetic joint infections. Arthroplast Today 2020;6:686-8.

18 Huang J, Zhang Y, Wang Z, et al. The serum level of D-dimer is not suitable for distinguishing between prosthetic joint infection and aseptic loosening. J Orthop Surg Res 2019;14:407.

19 Xiong L, Li S, Dai M. Comparison of D-dimer with CRP and ESR for diagnosis of periprosthetic joint infection. J Orthop Surg Res 2019;14:240.

20 Qin L, Li F, Gong X, et al. Combined measurement of D-dimer and C-reactive protein levels: highly accurate for diagnosing chronic periprosthetic joint infection. J Arthroplasty 2020;35:229-34.

21 Pannu TS, Villa JM, Patel PD, et al. The utility of serum D-dimer for the diagnosis of periprosthetic joint infection in revision total hip and knee arthroplasty. J Arthroplasty 2020;35:1692-5.

22 Pannu TS, Villa JM, Riesgo AM, et al. Letter to the editor on "combined measurement of D-dimer and C-reactive protein levels: highly accurate for diagnosing chronic periprosthetic joint infection". $J$ Arthroplasty 2021;36:e5.

23 Li C, Margaryan D, Ojeda-Thies C. Meta-Analysis of serum and/or plasma D-dimer in the diagnosis of periprosthetic joint infection. $J$ Orthop Surg Res 2020;15:298.

$24 \mathrm{Lu} \mathrm{G}$, Li T, Ye H, et al. D-Dimer in the diagnosis of periprosthetic joint infection: a systematic review and meta-analysis. J Orthop Surg Res 2020;15:265.

25 Wang Y, Man Z, Yuan T, et al. Reliability of D-dimer determination in diagnosis of Peri-Prosthetic joint infection: a systematic review and meta-analysis. Surg Infect 2021;22:374-82.

$26 \mathrm{Xu} \mathrm{H}$, Xie Jin-wei, Yang Jing-li, et al. Role of D-dimer and fibrinogen in the diagnosis of periprosthetic joint infection: a systematic review and Meta-Analysis. Orthop Surg 2021;13:692-700.

27 Fujimoto T, Kaneko T, Sunakawa T, et al. Elevation of fibrin degradation product (fdp) values prevents the negative conversion of serum CRP values after total knee arthroplasty. J Orthop 2018;15:940-4.

28 Parvizi J, Gehrke T, International Consensus Group on Periprosthetic Joint Infection. Definition of periprosthetic joint infection. $J$ Arthroplasty 2014;29:1331.

29 Proceedings of the International consensus meeting on periprosthetic joint infection. Foreword. J Orthop Res 2014;32 Suppl $1:$ S2-3.

30 Ottink KD, Wouthuyzen-Bakker M, Kampinga GA, et al. Puncture protocol in the diagnostic work-up of a suspected chronic prosthetic joint infection of the hip. J Arthroplasty 2018;33:1904-7. 
31 Schiffner E, Latz D, Thelen S, et al. Normal CRP and WBC values in total hip arthroplasty (THA) with signs of loosening. do we need a joint aspiration? J Clin Orthop Trauma 2019;10:566-70.

32 Shohat N, Goswami K, Fillingham Y, et al. Diagnosing periprosthetic joint infection in inflammatory arthritis: assumption is the enemy of true understanding. J Arthroplasty 2018;33:3561-6.

33 Mirza SZ, Richardson SS, Kahlenberg CA, et al. Diagnosing prosthetic joint infections in patients with inflammatory arthritis: a systematic literature review. J Arthroplasty 2019;34:1032-6.

34 Shahi A, Deirmengian C, Higuera C, et al. Premature therapeutic antimicrobial treatments can compromise the diagnosis of late periprosthetic joint infection. Clin Orthop Relat Res 2015;473:2244-9.

35 Ali F, Wilkinson JM, Cooper JR, et al. Accuracy of joint aspiration for the preoperative diagnosis of infection in total hip arthroplasty. $J$ Arthroplasty 2006;21:221-6. 